\title{
Incidence of new-onset atrial fibrillation in COVID-19 is associated with increased epicardial adipose tissue
}

\author{
Leandro Slipczuk ${ }^{1,2}$ - Francesco Castagna ${ }^{1} \cdot$ Alison Schonberger $^{3} \cdot$ Eitan Novogrodsky $^{3} \cdot$ Damini Dey $^{4}$. \\ Ulrich P. Jorde $^{1,2} \cdot$ Jeffrey M. Levsky ${ }^{2,3} \cdot$ Luigi Di Biase $^{1,2} \cdot$ Mario J. Garcia $^{1,2,3}$
}

Received: 27 April 2021 / Accepted: 24 June 2021 / Published online: 7 July 2021

(c) Springer Science+Business Media, LLC, part of Springer Nature 2021

\begin{abstract}
Purpose Coronary artery calcium (CAC) and epicardial adipose tissue (EAT) can predict AF in the general population. We aimed to determine if CAC and EAT measured by computed tomographic (CT) scanning can predict new-onset AF in patients admitted with COVID-19 disease.

Methods We performed a retrospective, post hoc analysis of all patients admitted to Montefiore Medical Center with a confirmed COVID-19 diagnosis from March 1st to June 23rd, 2020, who had a non-contrast CT of the chest within 5 years prior to admission. We determined ordinal CAC scores and quantified the EAT volume and examined their relationship with inpatient mortality.

Results A total of 379 patients were analyzed. There were 16 events of new-onset AF (4.22\%). Patients who developed AF during the index admission were more likely to be male ( 75 vs 47\%, $p<0.001)$ and had higher EAT (129.5 [76.3-197.3] vs 91.0 [60.0-129.0] ml, $p=0.049)$. There were no differences on age (68 [56-71] vs 68 [58-76] years; $p=0.712)$, BMI $(28.5$ [25.3-30.8] vs 26.9 [23.1-31.8] kg/m²; $p=0.283$ ), ordinal CAC score (3 [1-6] vs 2 [0-4]; $p=0.482$ ), or prevalence of diabetes $(56.3$ vs $60.1 \% ; p=0.761)$, hypertension $(75.0$ vs $87.3 \%, p=0.153)$, or coronary artery disease $(50.0$ vs $39.4 \%, p=0.396)$. Patients with new-onset AF had worse clinical outcomes (death/intubation/vasopressors) (87.5 vs $44.1 \% ; p=0.001$ ).

Conclusion Increased EAT measured by non-contrast chest CT identifies patients hospitalized with COVID-19 at higher risk of developing new-onset AF. Patients with new-onset AF have worse clinical outcomes.
\end{abstract}

Keywords COVID-19 $\cdot$ Coronary artery calcium $\cdot$ Epicardial adipose tissue $\cdot$ Atrial fibrillation

\section{Introduction}

Coronavirus disease (COVID-19), caused by the severe acute respiratory syndrome coronavirus 2 (SARS-CoV-2), has rapidly spread globally. Although most cases recover, COVID-19 still appears to retain an elevated mortality with the number of deaths escalating to over 2.5 million worldwide through March 2021 [1].

Leandro Slipczuk

1slipczukb@montefiore.org

1 Cardiology Division, Montefiore Medical Center, $111 \mathrm{E}$ 210th, Bronx, NY 10467, USA

2 Albert Einstein College of Medicine, Bronx, NY, USA

3 Radiology Division, Montefiore Medical Center, Bronx, NY, USA

4 Cedars-Sinai Medical Center, Los Angeles, CA, USA
Initial reports from China suggested an incidence of COVID-19 arrhythmias of 17\% with higher incidence in patients admitted to the ICU (up to 44\%) [2]. Further studies in different populations showed an incidence of new onset AF of around 4-8\% [3, 4]. Consequences of AF in COVID19 are still uncertain; some authors reported possible worse outcomes [5], whilst others did not [6].

Computed tomographic (CT) scanning can be used to detect and quantify visceral fat. In the past few years, epicardial adipose tissue (EAT) has attracted a lot of interest as an independent risk factor for new-onset AF, possibly due to pro-inflammatory cytokines and fibrosis of the atrial myocardium $[7,8]$. EAT is a metabolically active fat depot that makes up for approximately $20 \%$ of total heart weight, lies between serous epicardium and the fibrous pericardium, and can be quantified from non-gated non-contrast chest CT [9].

CT scanning can also identify the presence and extent of coronary atherosclerosis. The coronary artery calcification 
scoring (CAC) is an established and extensively validated imaging biomarker for cardiovascular risk, allowing enhanced risk reclassification for the prediction of all cause and cardiac specific mortality in asymptomatic subjects [10]. Moreover, $\mathrm{CAC}$ at a single time point and its progression have been associated with an increased risk for AF [11]. Although the traditional Agatston method for measuring CAC requires ECG-gated acquisition, good correlation has been demonstrated between CAC identified on non-gated CT scans and ordinal scores obtained from gated CT scans [12].

Our hypothesis was that EAT and/or CAC, as markers of a pro-inflammatory milieu and coronary atherosclerosis, respectively, are predictors of new-onset AF during COVID19 hospitalization. As many patients hospitalized with COVID-19 currently undergo chest CT examinations, EAT analysis could be readily available and provide additional clinical value for appropriate monitoring.

\section{Methods}

We conducted a retrospective, post hoc analysis of all patients who were admitted to Montefiore Medical Center with a confirmed COVID-19 diagnosis by polymerase chain reaction (PCR) from March 1st to June 23rd, 2020. Sample for RT-PCR was obtained by either nasopharyngeal or oropharyngeal swab. The follow-up period was from March 1st to June 26th, 2020. Patients who had a non-contrast CT of the chest at our facility after March 1st, 2015, constituted the main study cohort. Baseline demographic, clinical, and laboratory variables (including inflammatory biomarkers) were retrieved from our electronic medical record system. Baseline characteristics and outcomes were compared with patients who were admitted with COVID-19 but did not have a CT study performed. The investigators had direct access to primary data.

The study was approved by our Institutional Review Board (Office of Human Research Affairs at Albert Einstein College of Medicine).

\subsection{CT acquisition}

Patients were imaged on the following CT scanners without contrast administration or electrocardiographic gating: GE Optima 660, GE Lightspeed VCT, Neurologica Corp Caretom, Siemens Somatom Sensation 16, and GE Optima CT 540. Images were reconstructed at $2.5 \mathrm{~mm}$ slice thickness. Scans with reconstructions at different slice thickness were excluded.

\subsection{Coronary calcium score}

Two readers blinded from clinical data calculated ordinal scores from standard non-gated chest CT studies using the methods described by Shemesh et al. for patients without history of myocardial revascularization [12]. Briefly, the right coronary artery, left main, left anterior descending, and left circumflex arteries were each given a score of $0-3$ for presence and extent of calcium with 0 (none), $1(<1 / 3$ of the artery length calcified), 2 ( $\geq 1 / 3$ to $<2 / 3$ calcified), and 3 ( $\geq 2 / 3$ calcified). These scores were summed across the 4 arteries, providing a range of possible scores from 0 to 12 (see Fig. 1A and B). We calculated the inter-observer variability based on a random sample. Agreement for severity class was high (interclass correlation coefficient $=0.980$, $p<0.001)$.
A

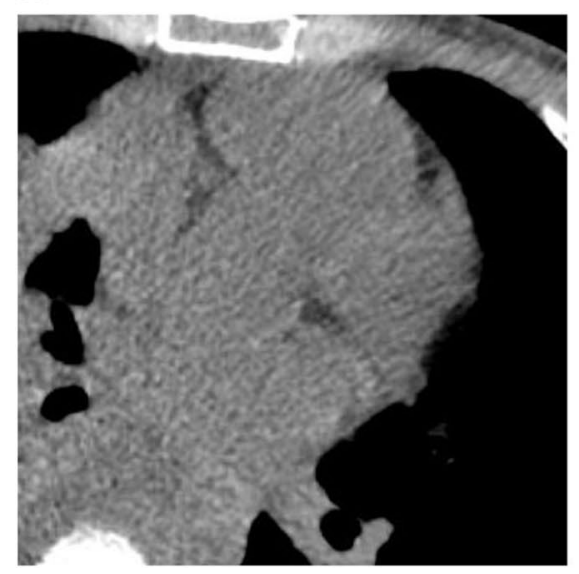

B

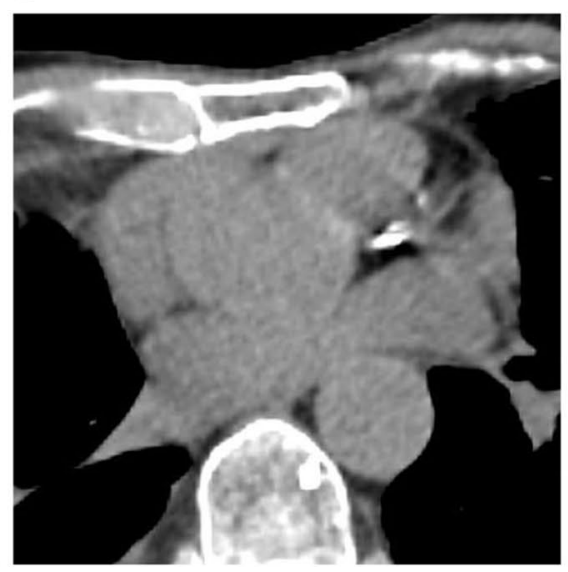

C

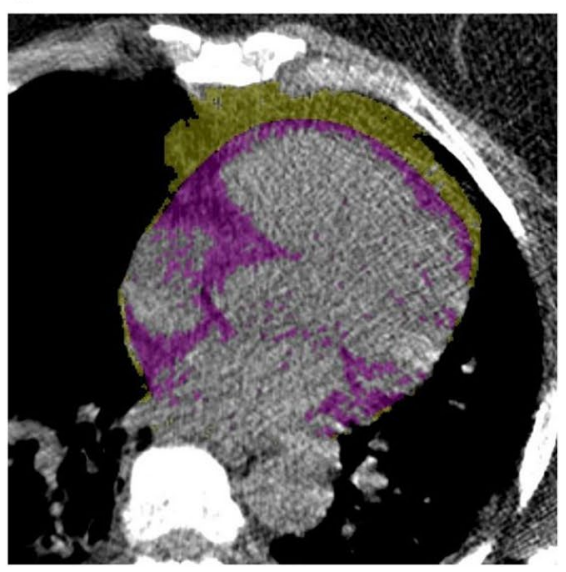

Fig. 1 Example of patients CTs and measurement of CAC and EAT. A Patient with CAC $=0$ who survived. B Patient with CAC $=9$ who died. C Patient with EAT $=138 \mathrm{ml}$ who died; EAT highlighted in purple by QFAT Software 


\subsection{Quantification of EAT}

For the measurement of EAT, we used QFAT software. As previously described [13], QFAT uses convolutional deep learning for fully automated quantification of epicardial and thoracic volumes. Accuracy and reproducibility of QFAT have been previously validated $[14,15]$. Contours were reviewed by an expert user and manually modified when needed. Superior and inferior limits of the pericardium were identified as the bifurcation of the pulmonary trunk and the posterior descending artery, respectively. EAT was defined as adipose tissue within the pericardium (see Fig. 1C). Patients with significant artifacts from ICDs or poor acquisition were excluded.

\subsection{Statistical analysis}

Continuous data are displayed as mean \pm standard deviation or median (25-75\% interquartile range) and compared with the Student's t-test, or Wilcoxon ranks-sum, as appropriate. Categorical data are presented as percent and compared by the chi-squared test. A binomial logistic regression was performed to ascertain the effect of CAC and EAT on the likelihood of developing AF. Kaplan-Meier survival analysis and log rank were conducted to compare the freedom from the composite event of intubation, pressor need, or death. June 26th, 2020, was the end of the censoring period. A $p<0.05$ was considered statistically significant. Statistical analysis was performed with SPSS (IBM Corp, ver. 25, Armonk, NY).

\section{Results}

\subsection{Study population and baseline characteristics}

From 4311 patients who were hospitalized at Montefiore between March 1st and June 23rd, 2020, with confirmed COVID-19 by PCR, we identified 504 patients who had a non-contrast chest CT performed during or within 5 years prior to admission. A total of 125 patients were excluded, 114 due to prior AF, and 11 due to uninterpretable CT. A total of 379 patients were finally included for analysis. Overall median age was 68 (IQR 58-76) years, $48.3 \%$ were male, and 39.3\% Hispanic. Diabetes (DM) (59.9\%), hyperlipidemia $(70.7 \%)$, and hypertension (86.8\%) were highly prevalent, whereas CAD and asthma/COPD were present in $<50 \%$ of patients (39.8 and 49.5\%, respectively). There was no difference on $\mathrm{CHA}_{2} \mathrm{DS}_{2}$-VASc score between the group without and with new-onset $\mathrm{AF}$ (4 [3-5] vs 3 [2-4]; $p=0.818$ ). On average, patients presented to the hospital 2 days after onset of symptoms (IQR 0-5). There were 16 episodes of newonset $\mathrm{AF}(4.22 \%)$ detected during hospitalization. Patients who developed new-onset AF were more likely to be male ( 75.0 vs $47.1 \% ; p=0.029$ ). There were no differences in age (68.0 [56.3-70.5] vs 68.0 [58.0-76.0] years; $p=0.712)$, BMI (28.5 [25.3-30.8] vs $\left.26.9[23.1-31.8] \mathrm{kg} / \mathrm{m}^{2} ; p=0.283\right)$, or prevalence of DM (56.3 vs $60.1 \% ; p=0.761)$, hyperlipidemia ( 87.5 vs $70.0 \% ; p=0.132)$, hypertension $(75.0$ vs $87.3 \% ; p=0.154)$, CAD (50.0 vs $39.4 \% ; p=0.396$ ), or asthma/COPD (37.5 vs $49.0 \%$; $p=0.366$ ). On presentation, there were no significant differences on vital signs (Table 1). There was a small difference on serum potassium levels (4.7 [4.4-5.7] vs 4.4 [4.0-4.9] mEq/1; $p=0.027)$ but not on other routine laboratory tests at the time of presentation. Patients who developed AF had worst outcomes with longer hospitalization (Fig. 2; 15 [8-26.8] vs 6 [4-12] days; $p<0.001$ ), requirement of pressors ( 75 vs $22 \%$; $p<0.0001$ ), and intubation ( 81.3 vs $22.0 \%$; $p<0.001$ ). Patients with newonset AF were more likely to receive IV steroids (56.3 vs $25.5 \% ; p=0.005)$ but there were no differences on use of inotropes ( 0.0 vs $1.4 \% ; p=0.637)$, hydroxychloroquine $(75.0$ vs $64.5 \% ; p=0.387)$, chloroquine $(0.0$ vs $1.9 \% ; p=0.575)$, azithromycin ( 37.5 vs $29.2 \% ; p=0.476$ ), or antibiotics ( 93.8 vs $80.2 \%$; $p=0.177)$.

When compared to the group admitted for COVID-19 within the same timeframe without a prior $\mathrm{CT}$ within 5 years $(n=3807)$, patients with no prior CT were younger $(65$ [54-77] vs 70 [60-77] years; $p<0.001$ ), had higher BMI (28.8 [24.9-33.9] vs $\left.27.3[23.3-31.9] \mathrm{kg} / \mathrm{m}^{2} ; p<0.001\right)$, and had lower prevalence of DM (51.9 vs $62.3 \% ; p<0.001)$, hyperlipidemia (55.0 vs $74.6 \% ; p<0.001)$, hypertension (69.5 vs $88.6 \%, p<0.001)$, CAD (23 vs $48.5 \%, p<0.001)$, and lung disease ( 25.2 vs $52.3 \%, p<0.001)$. There was no difference in gender ( 53.2 vs $49.5 \%$ male, $p=0.12)$. Incidence of new-AF for the cohort without prior CT was not significantly different when compared to the group without prior CT lower than one with prior CT (4 vs $5 \% ; p=0.987$ ). Our cohort also had no difference in mechanical ventilation ( 23.9 vs $21.5 \%$; $p=0.213$ ) but higher requirement for pressors ( 23.9 vs $19.1 \% ; p=0.012)$, and death rates (40.0 vs $28.2 \% ; p<0.001)$.

\subsection{Coronary artery calcium}

Ordinal CAC score was calculated in 379 patients. CAC $>0$ was present in 231 patients with 116 patients having mild (1-3), 72 moderate (4-6), and 43 severe (7-12) CAC with an overall population median CAC score of 2 (IQR 0.0-4.3). There was no difference in CAC score between the newonset and no AF groups (Fig. 3A; Ordinal CAC 2.5 [1.0-5.5] vs 2.0 [0-4.3]; $p=0.482$ ). On a ROC curve analysis, CAC showed an AUC of $0.551(p=0.494)$. Patients with CAC $\geq 4$ (moderate to severe) had no different incidence of new-AF (Fig. 3A OR $=0.959, p=0.939$ ) but had worse clinical outcomes compared to patients with $\mathrm{CAC}<4$, with higher 
Table 1 Baseline characteristics of patients admitted with COVID-19. Columns show the different demographic, clinical, laboratory, and CT values of the overall group (all), those who did not develop AF (no AF), and those who did (AF). $\mathrm{P}$ value compared no $\mathrm{AF}$ vs $\mathrm{AF}$ groups

\begin{tabular}{|c|c|c|c|c|c|}
\hline & $\begin{array}{l}\text { ALL } \\
n=379\end{array}$ & $\begin{array}{l}\text { No AF } \\
n=363\end{array}$ & $\begin{array}{l}\mathrm{AF} \\
n=16\end{array}$ & $\mathrm{p}$ & \\
\hline \multicolumn{6}{|l|}{ Demographics } \\
\hline Age, years & $n=379$ & $68(58-76)$ & $68(58-76)$ & $68(56.3-70.5)$ & 0.712 \\
\hline BMI $\left(\mathrm{kg} / \mathrm{m}^{2}\right)$ & $n=368$ & $26.9(23.3-31.7)$ & $26.9(23.1-31.8)$ & $28.5(25.3-30.8)$ & 0.283 \\
\hline Male gender, no (\%) & $n=379$ & $183 / 379(48.3)$ & $171 / 363(47.1)$ & $12 / 16(75)$ & 0.029 \\
\hline \multicolumn{6}{|l|}{ PMH } \\
\hline Diabetes, no (\%) & $n=379$ & $227 / 379(59.9)$ & $218 / 363(60.1)$ & $9 / 16(56.3)$ & 0.761 \\
\hline Hyperlipidemia, no (\%) & $n=379$ & $268 / 379(70.7)$ & $254 / 363(70.0)$ & $14 / 16(87.5)$ & 0.132 \\
\hline Hypertension, no (\%) & $n=379$ & $329 / 379(86.8)$ & $317 / 363(87.3)$ & $12 / 16(75)$ & 0.154 \\
\hline Asthma/COPD, no (\%) & $n=379$ & $184 / 379(49.0)$ & $178 / 363(49)$ & $6 / 16(37.5)$ & 0.366 \\
\hline Coronary artery disease, no (\%) & $n=379$ & $151 / 379(39.8)$ & $143 / 363(39.4)$ & $8 / 16(50)$ & 0.396 \\
\hline \multicolumn{6}{|l|}{ Presentation } \\
\hline Symptom duration, days & $n=365$ & $2(0-5)$ & $2(0-5)$ & $3(0.3-3.8)$ & 0.927 \\
\hline Temperature, F & $n=376$ & $98.6(98.0-99.7)$ & $98.6(98.0-99.7)$ & $98.9(98.6-100.1)$ & 0.093 \\
\hline Systolic BP, mmHg & $n=378$ & $132(112-150)$ & $131(111-149)$ & $141(118-168)$ & 0.104 \\
\hline Diastolic BP, mmHg & $n=377$ & $73(63-84)$ & $73(63-84)$ & $75(66-83)$ & 0.769 \\
\hline $\mathrm{HR}, \mathrm{bpm}$ & $n=377$ & $95(82-109)$ & $95(83-109)$ & $87(77-109)$ & 0.412 \\
\hline Pulse oximeter saturation, $\%$ & $n=376$ & $96(92-98)$ & $96(92-98)$ & $94(89-96)$ & 0.062 \\
\hline Respiratory rate, bpm & $n=376$ & $20(18-22)$ & $20(18-22)$ & $22(18-27)$ & 0.115 \\
\hline WBC count, $\mathrm{k} / \mu \mathrm{L}$ & $n=371$ & $7.3(5.0-11.0)$ & $7.3(5.0-11.0)$ & $7.3(5.0-11.8)$ & 0.926 \\
\hline Hemoglobin, g/dL & $n=371$ & $12.0(10.3-13.4)$ & $11.9(10.3-13.5)$ & $12.1(10.6-13.0)$ & 0.568 \\
\hline Sodium, mEq/L & $n=368$ & $137(134-141)$ & $137(134-141)$ & $137(134-142)$ & 0.858 \\
\hline Potassium, mEq/L & $n=359$ & $4.4(4.0-4.9)$ & $4.4(4.0-4.9)$ & $4.7(4.4-5.7)$ & 0.027 \\
\hline EGFR, $\mathrm{mL} / \mathrm{min} / \mathrm{BSA}$ & $n=369$ & $51.8(21.9-80.2)$ & $52.3(21.2-80.3)$ & $44.8(27.4-75.2)$ & 0.927 \\
\hline Glucose & $n=369$ & $133(106.5-195.5)$ & $130(105.5-196.0)$ & $155(134.8-195.0)$ & 0.214 \\
\hline Lactic acid, mmol/L & $n=161$ & $2(1.4-2.9)$ & $2(1.4-2.9)$ & $1.8(1.5-3.5)$ & 0.692 \\
\hline ProBNP, pg/mL & $n=193$ & $676(174-2956)$ & $656(158-3025)$ & $1267(509-8857)$ & 0.217 \\
\hline D-dimer, $\mu \mathrm{g} / \mathrm{mL}$ & $n=147$ & $2.5(1.2-4.3)$ & $2.4(1.1-4.2)$ & $2.7(2.1-20)$ & 0.304 \\
\hline C-reactive protein, $\mu \mathrm{g} / \mathrm{mL}$ & $n=169$ & $11.5(4.4-21.8)$ & $11.3(4.6-21.2)$ & $17.1(3.4-29.4)$ & 0.653 \\
\hline Fibrinogen, mg/dL & $n=226$ & $642(516-745)$ & $644(516-745)$ & $517(481-x x)$ & 0.245 \\
\hline LDH, U/L & $n=228$ & $373(280-502)$ & $372(278-497)$ & $376(285-528)$ & 0.694 \\
\hline Ferritin, ng/mL & $n=131$ & $770(353-1555)$ & $755(353-1432)$ & $1991(649-4337)$ & 0.177 \\
\hline Troponin T, ng/mL & $n=304$ & $0.01(0.01-0.05)$ & $0.01(0.01-0.05)$ & $0.01(0.01-0.04)$ & 0.511 \\
\hline \multicolumn{6}{|l|}{ Medications during admission } \\
\hline Hydroxychloroquine, no (\%) & $n=379$ & $246 / 379(64.9)$ & $234 / 363(64.5)$ & $12 / 16(75.0)$ & 0.387 \\
\hline Azithromycin, no (\%) & $n=379$ & $112 / 379(29.6)$ & $106 / 363(29.2)$ & $6 / 16(37.5)$ & 0.476 \\
\hline Other antibiotics, no (\%) & $n=379$ & $306 / 379(80.7)$ & $291 / 363(24.5)$ & 15/16 (93.8) & 0.177 \\
\hline IV steroids, no (\%) & $n=379$ & 98/379 (25.9) & $89 / 363(24.5)$ & $9 / 16(53.3)$ & 0.005 \\
\hline Chloroquine, no (\%) & $n=379$ & $7 / 379(1.8)$ & $7 / 363(1.9)$ & $0 / 16(0)$ & 0.575 \\
\hline ARBS, no (\%) & $n=379$ & $20 / 379(5.3)$ & 20/363 (5.5) & $0 / 16(0)$ & 0.335 \\
\hline Statin, no $(\%)$ & $n=379$ & $145 / 379(38.3)$ & $137 / 363(37.7)$ & $8 / 16(50)$ & 0.323 \\
\hline \multicolumn{6}{|l|}{ Clinical outcomes } \\
\hline Intubation & $n=379$ & $93 / 379(25)$ & $80 / 363(22)$ & $13 / 16(81)$ & $<0.001$ \\
\hline Mortality & $n=379$ & $145 / 379(38.3)$ & $136 / 363(37.5)$ & $9 / 16(56.3)$ & 0.130 \\
\hline Pressors & $n=379$ & $92 / 379(24.3)$ & $80 / 363(22)$ & $12 / 16(75)$ & $<0.001$ \\
\hline Inotropes & $n=379$ & $5 / 379(1.3)$ & $5 / 363(1.4)$ & 0/16 (0) & 0.637 \\
\hline \multicolumn{6}{|l|}{ CAC and EAT } \\
\hline $\mathrm{CAC}$ & $n=358$ & $2.0(0-4.3)$ & $2.0(0-4.3)$ & $2.5(1-5.5)$ & 0.482 \\
\hline Epicardial fat, mL & $n=359$ & $92(60-130)$ & $91(60-129)$ & $130(76-197)$ & 0.049 \\
\hline
\end{tabular}

Statistical significance highlighted in bold

$A L T$ alanine transaminase; $A S T$ aspartate transaminase; $B M I$ body mass index; $B P$ blood pressure; $B U N$ blood urinary nitrogen; $C A C$ ordinal coronary artery calcium; $C A D$ coronary artery disease; $C O P D$ chronic obstructive pulmonary disease; $E G F R$ estimated glomerular filtration rate; $F$ Fahrenheit; $H R$ heart rate; $I L-6$ interleukin 6; $I V$ intravenous; $L D H$ lactate dehydrogenase; proBNP ProB-type natriuretic peptide; $W B C$ white blood cell 
Fig. 2 Clinical outcomes for new-onset AF. Kaplan-Meir curve shows freedom from intubation, pressor, and death in days according to new $\mathrm{AF}$ (red) vs without new AF (blue). $P=0.028$

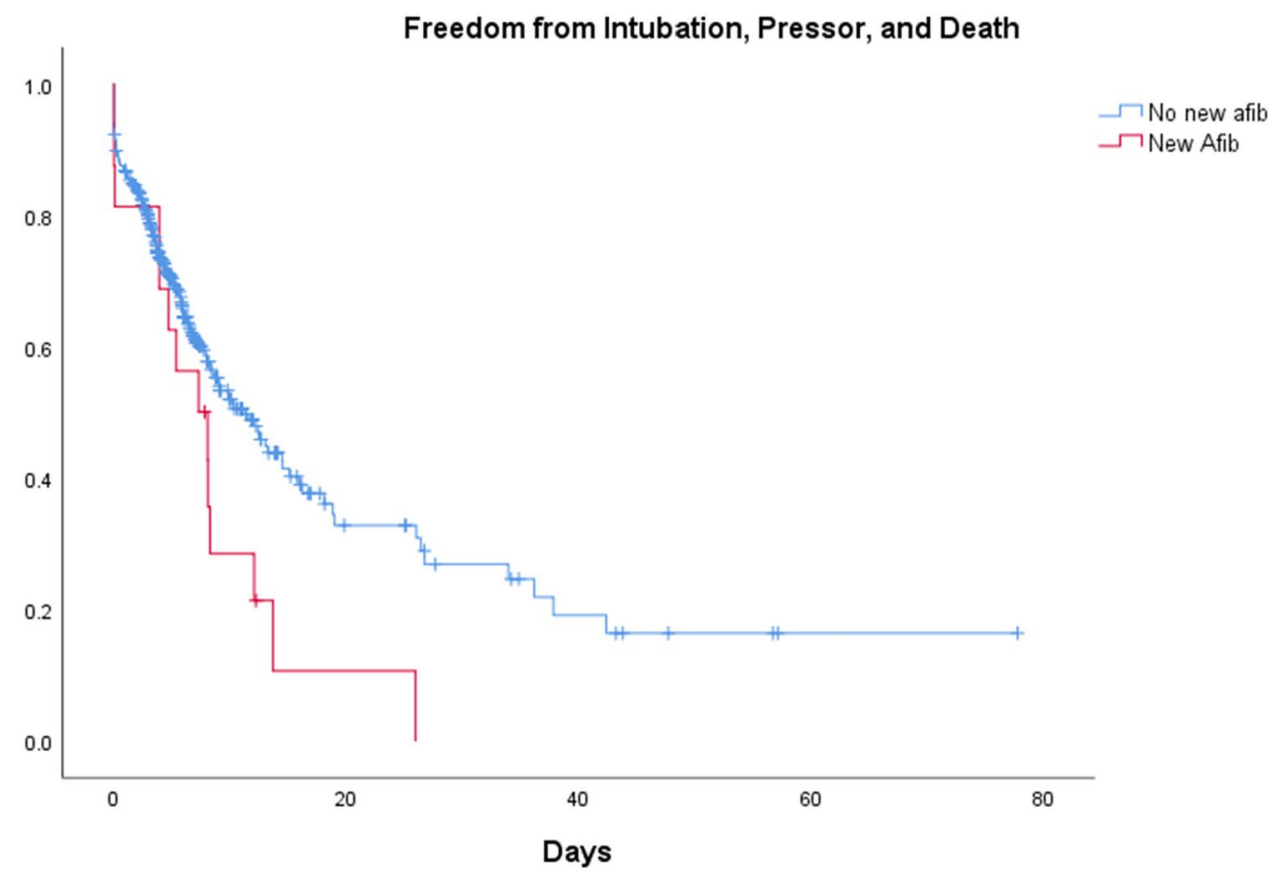

A CAC B Epicardial Fat tors tors of COVID-19 new-onset EAT (B) in the group without new-onset AF (black) and newonset AF (gray) during COVID19 hospitalization. $*=p<0.05$

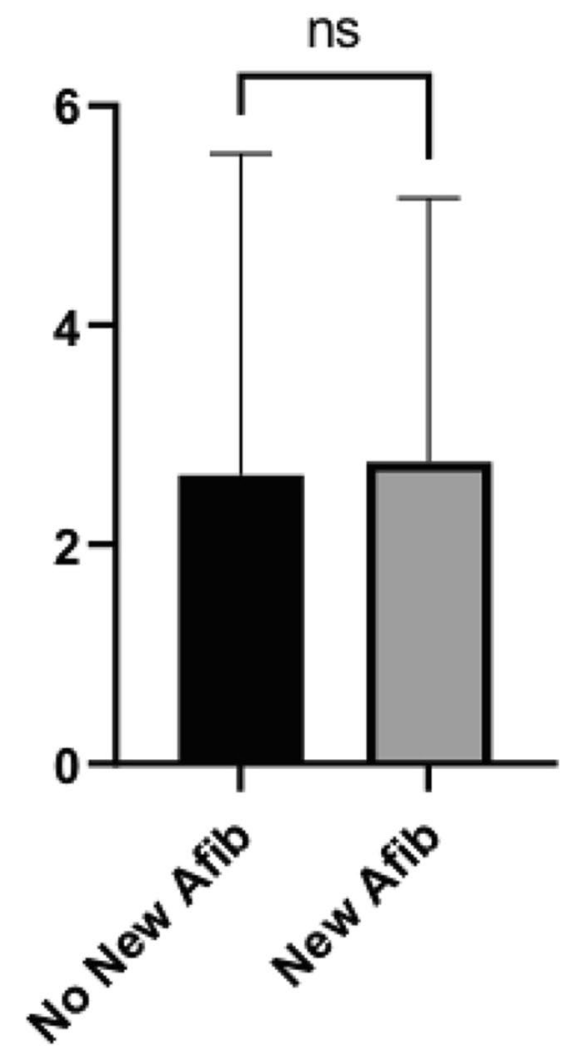


composite intubation/pressor/death (Fig. 4A; 59.1\% vs 39.9; $p=0.001)$.

\subsection{Epicardial adipose tissue}

EAT was calculated in 357 patients. Median volume in the overall population was $92 \mathrm{ml}(60-130 \mathrm{ml})$. Patients who developed Afib had a higher EAT compared to the patients who did not (Fig. 3B and C; 129.5 [76.3-197.3] vs $91.0 \mathrm{ml}$ [60.0-129.0]; $p<0.05$ ). For each $\mathrm{ml}$ in EAT, new-onset AF HR increased by $0.8(p=0.024)$. On a ROC curve analysis, EAT showed an AUC of $0.655(p=0.049)$. Patients with EAT $\geq$ median had worse outcomes with higher intubation/pressor/death (Fig. 4B; 129.5 [76.3-197.3] vs $91.0 \mathrm{ml}$ [60.0-129.0]; $p<0.05)$.

\section{Discussion}

The main finding in our study is the association of EAT with new-onset $\mathrm{AF}$ in a large cohort of patients admitted with COVID-19. Moreover, in our study, patients with new-onset AF showed worse clinical outcomes.

To our knowledge, this represents the first study to show an association of EAT with new-onset AF in COVID-19 patients. We showed that the group with new-onset AF had worse clinical outcomes with higher composite of intubation/pressors/death (Fig. 2) and higher EAT (Fig. 3B). Also, for each $\mathrm{ml}$ increase in EAT, new-onset AF increased by $0.8 \%$. On a regression model, EAT $>$ median has a $\mathrm{HR}=3.8$, $p=0.042$. Moreover, both higher CAC and EAT predicted worse clinical outcomes (Fig. 4). A number of published papers have investigated the relation between AF and clinical outcomes in patients with COVID-19; however, the results have not been consistent. Our results show that new-onset $\mathrm{AF}$ in particular could also be linked to adverse outcomes.

Population studies have established increased body mass index as a predictor of AF [16]. However, heterogeneity of fat distribution could explain differences in AF within same BMI [17]. It has been demonstrated that EAT is a metabolically active fat with its own ganglionated plexi. Multiple studies have found a prognostic role of EAT for new-onset, persistent AF, and clinical outcomes. A meta-analysis by Wong et al. involving 352,275 individuals found a strong and graded association with each increase of 1-SD associated with 2.6-fold higher odds of AF [18]. Moreover, the association was greater than for measures of abdominal or overall adiposity. Possible mechanisms include fatty infiltration [19], atrial myocardial fibrosis (possibly through secretion of TGF- $\beta$ ) [20], local inflammation [21], oxidative stress [22], adipocyte-related gene expression [23], autonomic dysfunction [24], and diastolic dysfunction [25].

Gauss et al. found a significant association between CAC, EAT, and systemic inflammatory markers [26]. Severity of COVID-19 infection has been associated to an immune dysregulation. In particular, monocytes from COVID-19 patients have sustained expression of the cytokines tumor necrosis factor (TNF) and interleukin-6 (IL-6) [27]. Mazurek et al. found that EAT is a source of several inflammatory mediators in high-risk cardiac patients with local expression of monocyte chemotactic protein (MCP)-1, IL-6, and TNF- $\alpha$ [28]. Moreover, ACE2 internalization after binding with COVID-19 results in a reduction of ACE2 in the cell surface. Loss of ACE2 promotes EAT inflammation, pericarditis, and the development of pericardial effusion, all of which may predispose to the development of AF [29].

It is also known that baseline pro-inflammatory risk factors (such as diabetes, hypertension, CAD, and COPD)
A

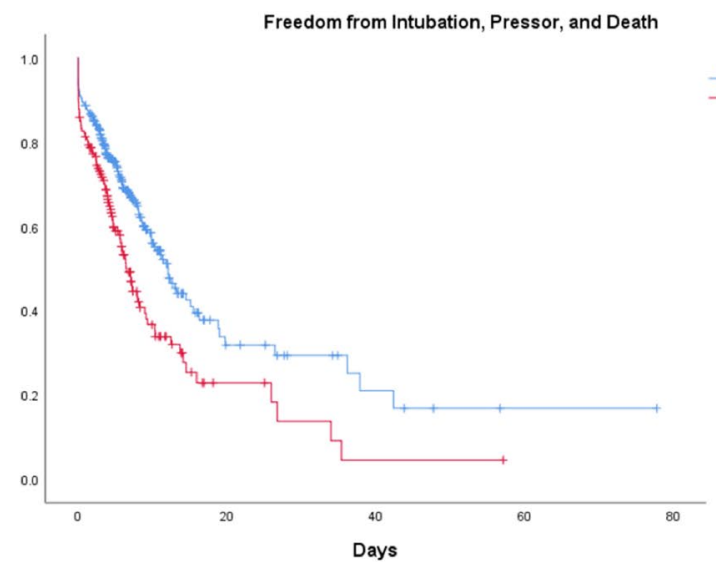

B

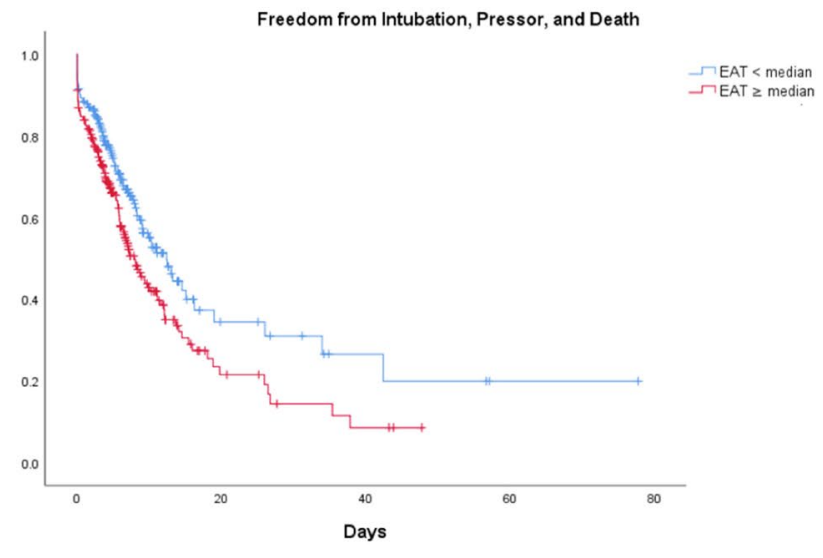

Fig. 4 Clinical outcomes for CAC $\geq 4$, EAT $\geq$ median. Kaplan-Meier curves show freedom from intubation/pressor/death in days according to CAC score (A) and EAT (B). $p=0.001$ for both 
exacerbate the cytokine storm related to severe cases of COVID-19 [30]. We showed here that EAT, possibly through a pro-inflammatory local response, has predictive value of new-onset AF in patients admitted with COVID-19.

The most accurate imaging technique to assess epicardial fat burden is volumetric quantification [17]. Measurement of EAT by CT has represented in the past a cumbersome process limiting its clinical applicability. Fully automated quantification by QFAT, through the use of a convolutional neural network approach, brings measurement process to a clinically useful and readily available tool with an average quantification time of $1.57 \mathrm{~s}$ [15].

Data from multiple cohorts shows that CAC effectively stratifies patients for risk of long-term all-cause and cardiovascular mortality better than traditional risk-factors $[10$, 31-33]. Likewise, data from MESA by O'Neal et al. showed that CAC predict incidence of AF [11].

The pathophysiologic link between CAC and AF remains unclear. One possibility is through their common association with cardiovascular risk factors. Imaging studies have shown that patients with higher CAC usually have larger atria and pulmonary veins, anatomical findings which have been associated with development of AF. In the MESA study, presence and progression of CAC seem to be independent predictors of AF [34]. Higher levels of inflammatory biomarkers have also been associated with development of CAC [35] and AF [36]. In our study, however, we did not find an association between CAC and new-onset AF in COVID-19 hospitalized patients. It is possible that the association between $\mathrm{AF}$ and coronary atherosclerosis is established over long term and is not relevant in acutely ill patients with respiratory failure.

Moreover, van der Hooft et al. have described an association between high-dose corticosteroids and development of AF [37]. Even though the difference in corticosteroids between groups in our study was small, it is possible that this factor played an additional role in new-onset AF. Additionally, $\mathrm{CHA}_{2} \mathrm{DS}_{2}$ VASc score has been associated with worse outcomes in COVID-19 [38]. We did not find an association between $\mathrm{CHA}_{2} \mathrm{DS}_{2}$-VASc score and new-onset AF.

Lastly, it has been suggested that the incidence and associated increase in mortality are not specific to COVID-19 but rather a generalized response to the systemic inflammation of severe viral illnesses [4]. It is therefore possible that our findings could be similarly present in other severe viral illnesses.

\section{Limitations}

The inclusion of only patients who had a prior chest CT selected a higher risk population; however, the incidence of new-AF was similar to those without prior CT. Many inflammatory markers were not routinely collected at the beginning of the pandemic and we cannot therefore evaluate their interaction. We did not find an association with the development of AF during COVID-19 admission and CAC. Possible explanations may be the older population in our study given that association in MESA was stronger in younger patients $<62$ years of age, and differences in pathophysiologic triggers in patients with acute respiratory failure. Moreover, new-onset AF may be underestimated as not all patient had telemetry monitoring. Finally, our cohort was collected during the earliest period of the COVID-19 pandemic, during which limited therapeutic strategies most likely contributed to worse outcomes.

\section{Conclusion}

Increased EAT measured by non-contrast chest $\mathrm{CT}$ identifies patients hospitalized with COVID-19 at higher risk of developing new-onset AF. Patients with new-onset AF have worse clinical outcomes. Since a significant number of patients hospitalized with COVID-19 currently undergo chest CT examinations, EAT analysis could be readily available and provide additional clinical value.

Funding Dr. Dey is partly supported by NIH/NHLBI grant R01HL133616.

\section{Declarations}

Ethics approval The study was approved by our Institutional Review Board (Office of Human Research Affairs at Albert Einstein College of Medicine).

Conflict of interest The authors declare no competing interests.

\section{References}

1. Johns Hopkins University. Mortality analyses - Johns Hopkins Coronavirus Resource Center. Johns Hopkins Coronavirus Resource Center. 2020.

2. Wang D, Hu B, Hu C, Zhu F, Liu X, Zhang J, ... Peng Z. Clinical characteristics of 138 hospitalized patients with 2019 novel coronavirus-infected pneumonia in Wuhan, China. JAMA - J Am Med Assoc. 2020. https://doi.org/10.1001/jama.2020.1585.

3. Bhatla A, Mayer MM, Adusumalli S, Hyman MC, Oh E, Tierney A, ... Deo R. COVID-19 and cardiac arrhythmias. Heart Rhythm. 2020. https://doi.org/10.1016/j.hrthm.2020.06.016.

4. Musikantow D, Turagam M, Sartori S, Chu E, Kawamura I, Shivamurthy $\mathrm{P}, \ldots$ Reddy V. Atrial fibrillation in patients hospitalized with COVID-19: incidence, predictors, outcomes and comparison to influenza. JACC Clin Electrophysiology. 2021. https://doi.org/ 10.1016/j.jacep.2021.02.009.

5. Pardo Sanz A, SalidoTahoces L, Ortega Pérez R, González Ferrer E, Sanchez Recalde Á, Zamorano Gómez JL. New-onset atrial 
fibrillation during COVID-19 infection predicts poor prognosis. Cardiol J. 2020. https://doi.org/10.5603/cj.a2020.0145.

6. Izurieta HS, Graham DJ, Jiao Y, Hu M, Lu Y, Wu Y, ... Forshee R. Natural history of COVID-19: Risk factors for hospitalizations and deaths among $>26$ million U.S. Medicare beneficiaries. J Infect Dis. 2020. https://doi.org/10.1093/infdis/jiaa767.

7. Dey D, Nakazato R, Li D, Berman DS. Epicardial and thoracic fat - noninvasive measurement and clinical implications. Cardiovasc Diagn Ther. 2012. https://doi.org/10.3978/j.issn.2223-3652.2012. 04.03 .

8. Al Chekakie MO, Welles CC, Metoyer R, Ibrahim A, Shapira AR, Cytron J, ... Akar JG. Pericardial fat is independently associated with human atrial fibrillation. J Am Coll Cardiol. 2010. https:// doi.org/10.1016/j.jacc.2010.03.071.

9. Nagayama Y, Nakamura N, Itatani R, Oda S, Kusunoki S, Takahashi $\mathrm{H}$, ... Yamashita Y. Epicardial fat volume measured on nongated chest $\mathrm{CT}$ is a predictor of coronary artery disease. Eur Radiol. 2019. https://doi.org/10.1007/s00330-019-06079-x.

10. Greenland P, LaBree L, Azen SP, Doherty TM, Detrano RC. Coronary artery calcium score combined with Framingham score for risk prediction in asymptomatic individuals. J Am Med Assoc. 2004. https://doi.org/10.1001/jama.291.2.210.

11. O'Neal WT, Efird JT, Dawood FZ, Yeboah J, Alonso A, Heckbert SR, Soliman EZ. Coronary artery calcium and risk of atrial fibrillation (from the multi-ethnic study of atherosclerosis). Am J Cardiol. 2014. https://doi.org/10.1016/j.amjcard.2014.09.005.

12. Shemesh J, Henschke CI, Shaham D, Yip R, Farooqi AO, Cham $\mathrm{MD}, \ldots$ Yankelevitz DF. Ordinal scoring of coronary artery calcifications on low-dose CT scans of the chest is predictive of death from cardiovascular disease. Radiology. 2010. https://doi.org/10. 1148/radiol.10100383.

13. Otaki Y, Rajani R, Cheng VY, Gransar H, Nakanishi R, Shmilovich H, ... Dey D. The relationship between epicardial fat volume and incident coronary artery calcium. J Cardiovasc Comput Tomogr. 2011. https://doi.org/10.1016/j.jcct.2011.06.007.

14. Nakazato R, Shmilovich H, Tamarappoo BK, Cheng VY, Slomka PJ, Berman DS, Dey D. Interscan reproducibility of computeraided epicardial and thoracic fat measurement from noncontrast cardiac CT. J Cardiovasc Comput Tomogr. 2011. https://doi.org/ 10.1016/j.jcct.2011.03.009.

15. Commandeur F, Goeller M, Betancur J, Cadet S, Doris M, Chen $X, \ldots$ Dey D. Deep learning for quantification of epicardial and thoracic adipose tissue from non-contrast CT. IEEE Trans Med Imaging. 2018. https://doi.org/10.1109/TMI.2018.2804799.

16. Wang TJ, Parise H, Levy D, D’Agostino RB, Wolf PA, Vasan RS, Benjamin EJ. Obesity and the risk of new-onset atrial fibrillation. J Am Med Assoc. 2004. https://doi.org/10.1001/jama.292. 20.2471 .

17. Wong CX, Ganesan AN, Selvanayagam JB. Epicardial fat and atrial fibrillation: current evidence, potential mechanisms, clinical implications, and future directions. Eur Heart J. 2017. https://doi. org/10.1093/eurheartj/ehw045.

18. Wong CX, Sun MT, Odutayo A, Emdin CA, Mahajan R, Lau DH, ... Clarke R. Associations of epicardial, abdominal, and overall adiposity with atrial fibrillation. Circ Arrhythm Electrophysiol. 2016. https://doi.org/10.1161/CIRCEP.116.004378.

19. Mahajan R, Lau DH, Brooks AG, Shipp NJ, Manavis J, Wood JPM, ... Sanders P. Electrophysiological, electroanatomical, and structural remodeling of the atria as consequences of sustained obesity. J Am Coll Cardiol. 2015. https://doi.org/10.1016/j.jacc. 2015.04.058

20. Venteclef N, Guglielmi V, Balse E, Gaborit B, Cotillard A, Atassi $\mathrm{F}, \ldots$ Hatem SN. Human epicardial adipose tissue induces fibrosis of the atrial myocardium through the secretion of adipo-fibrokines. Eur Heart J. 2015. https://doi.org/10.1093/eurheartj/eht099.
21. Mazurek T, Kiliszek M, Kobylecka M, Skubisz-Głuchowska J, Kochman J, Filipiak K, ... Opolski G. Relation of proinflammatory activity of epicardial adipose tissue to the occurrence of atrial fibrillation. Am J Cardiol. 2014. https://doi.org/10.1016/j.amjca rd.2014.02.005.

22. Carnes CA, Chung MK, Nakayama T, Nakayama H, Baliga RS, Piao S, ... Van Wagoner DR. Ascorbate attenuates atrial pacinginduced peroxynitrite formation and electrical remodeling and decreases the incidence of postoperative atrial fibrillation. Circ Res. 2001. https://doi.org/10.1161/hh1801.097644.

23. Chilukoti RK, Giese A, Malenke W, Homuth G, Bukowska A, Goette A, ... Wolke C. Atrial fibrillation and rapid acute pacing regulate adipocyte/adipositas-related gene expression in the atria. Int J Cardiol. 2015. https://doi.org/10.1016/j.ijcard.2015.03.072.

24. Pokushalov E, Kozlov B, Romanov A, Strelnikov A, Bayramova S, Sergeevichev D, ... Steinberg JS. Long-Term suppression of atrial fibrillation by botulinum toxin injection into epicardial fat pads in patients undergoing cardiac surgery: one-year follow-up of a randomized pilot study. Circ Arrhythm Electrophysiol. 2015. https://doi.org/10.1161/CIRCEP.115.003199.

25. Fox CS, Gona P, Hoffmann U, Porter SA, Salton CJ, Massaro JM, ... Manning WJ. Pericardial fat, intrathoracic fat, and measures of left ventricular structure and function. Circulation. 2009. https:// doi.org/10.1161/CIRCULATIONAHA.108.828970.

26. Gauss S, Klinghammer L, Steinhoff A, Raaz-Schrauder D, Marwan $M$, Achenbach S, Garlichs CD. Association of systemic inflammation with epicardial fat and coronary artery calcification. Inflamm Res. 2015. https://doi.org/10.1007/s00011-015-0809-x.

27. Giamarellos-Bourboulis EJ, Netea MG, Rovina N, Akinosoglou K, Antoniadou A, Antonakos N, ... Koutsoukou A. Complex immune dysregulation in COVID-19 patients with severe respiratory failure. Cell Host Microbe. 2020. https://doi.org/10.1016/j. chom.2020.04.009.

28. Mazurek T, Zhang LF, Zalewski A, Mannion JD, Diehl JT, Arafat $\mathrm{H}, \ldots$ Shi Y. Human epicardial adipose tissue is a source of inflammatory mediators. Circulation. 2003. https://doi.org/10. 1161/01.CIR.0000099542.57313.C5.

29. Gawałko M, Kapłon-Cieślicka A, Hohl M, Dobrev D, Linz D. COVID-19 associated atrial fibrillation: incidence, putative mechanisms and potential clinical implications. IJC Heart Vasc. 2020. https://doi.org/10.1016/j.ijcha.2020.100631.

30. Huang C, Wang Y, Li X, Ren L, Zhao J, Hu Y, ... Cao B. Clinical features of patients infected with 2019 novel coronavirus in Wuhan, China. Lancet. 2020. https://doi.org/10.1016/S01406736(20)30183-5.

31. Nasir K, Rubin J, Blaha MJ, Shaw LJ, Blankstein R, Rivera JJ, ... Budoff MJ. Interplay of coronary artery calcification and traditional risk factors for the prediction of all-cause mortality in asymptomatic individuals. Circ Cardiovasc Imaging. 2012. https:// doi.org/10.1161/CIRCIMAGING.111.964528.

32. Budoff MJ, Shaw LJ, Liu ST, Weinstein SR, Mosler TP, Tseng PH, ... Berman DS. Long-Term prognosis associated with coronary calcification. Observations from a registry of 25,253 patients. $\mathbf{J}$ Am Coll Cardiol. 2007. https://doi.org/10.1016/j.jacc.2006.10. 079.

33. Knapper JT, Khosa F, Blaha MJ, Lebeis TA, Kay J, Sandesara $\mathrm{PB}, . .$. Shaw LJ. Coronary calcium scoring for long-term mortality prediction in patients with and without a family history of coronary disease. Heart. 2016. https://doi.org/10.1136/heart jnl-2015-308429.

34. O'Neal WT, Efird JT, Qureshi WT, Yeboah J, Alonso A, Heckbert SR, ... Soliman EZ. Coronary artery calcium progression and atrial fibrillation: the multi-ethnic study of atherosclerosis. Circ Cardiovasc Imaging. 2015. https://doi.org/10.1161/CIRCIMAGING.115.003786. 
35. Jenny NS, Brown ER, Detrano R, Folsom AR, Saad MF, Shea S, ... Jacobs DR. Associations of inflammatory markers with coronary artery calcification: results from the Multi-Ethnic Study of Atherosclerosis. Atherosclerosis. 2010. https://doi.org/10.1016/j. atherosclerosis.2009.08.037.

36. Aviles RJ, Martin DO, Apperson-Hansen C, Houghtaling PL, Rautaharju P, Kronmal RA, ... Chung MK. Inflammation as a risk factor for atrial fibrillation. Circulation. 2003. https://doi.org/ 10.1161/01.CIR.0000103131.70301.4F.

37. Van Der Hooft CS, Heeringa J, Brusselle GG, Hofman A, Witteman JCM, Kingma JH, ... Stricker BHC. Corticosteroids and the risk of atrial fibrillation. Arch Intern Med. 2006. https://doi.org/ 10.1001/archinte.166.9.1016.

38. Uribarri A, Núñez-Gil IJ, Aparisi Á, Arroyo-Espliguero R, Maroun Eid C, Romero R, ... Macaya C. Atrial fibrillation in patients with COVID-19. Usefulness of the CHA2DS2-VASc score: an analysis of the international HOPE COVID-19 registry. Rev Esp Cardiol (English Edition). 2021. https://doi.org/10. 1016/j.rec.2020.12.009.

Publisher's note Springer Nature remains neutral with regard to jurisdictional claims in published maps and institutional affiliations. 